\title{
Ketamine for acute catatonia: A case report
}

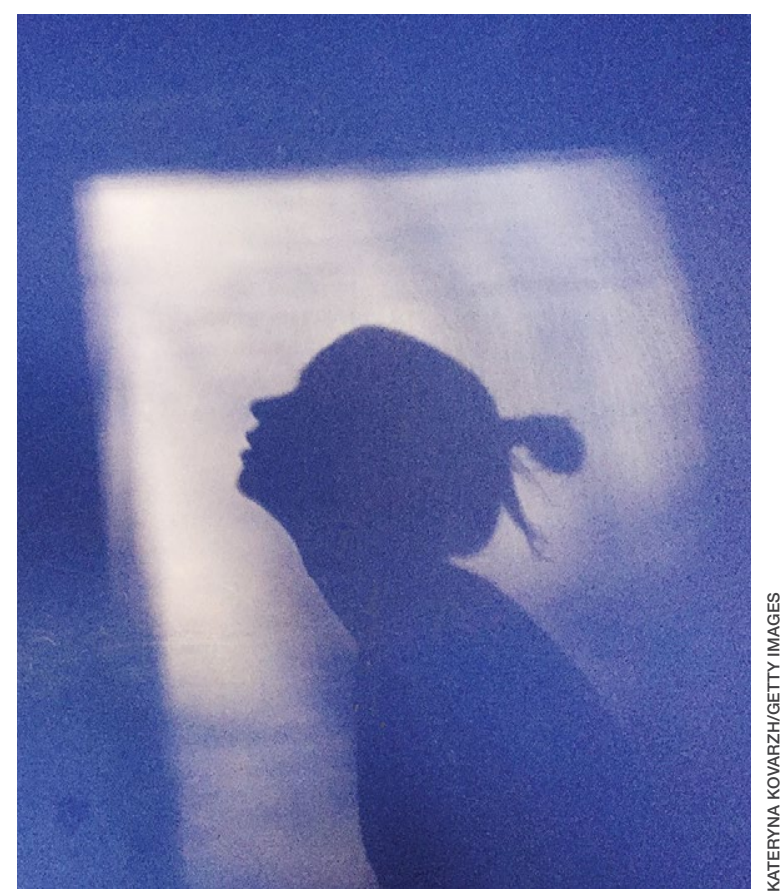

\section{Brian Kobayashi, BS}

Medical Student

UC Irvine School of Medicine Irvine, California

\section{Chela Wright, MD}

PGY-3 Psychiatry Resident Department of Psychiatry UC Irvine School of Medicine Irvine, California
Michael Burns, MD

Emergency Medicine Physician Department of Emergency Medicine

UC Irvine School of Medicine Irvine, California

\section{Rimal Bera, MD}

Clinical Professor of Psychiatry Department of Psychiatry UC Irvine School of Medicine Irvine, California

\section{Our patient's experience suggests this agent might reduce mutism and immobility}

$\mathrm{M}$ s. C, age 44, who has major depressive disorder (MDD), anxiety, obsessive-compulsive disorder (OCD) (religious subtype), and has experienced multiple episodes of treatment-resistant catatonia, is brought to the emergency department (ED) by her parents. She has immobility, mutism, rigidity, and decreased oral intake that she has experienced for 1 day.

The night before, Ms. C had been stressed about an upcoming job interview. She cancelled the interview and went to her bedroom. Later that night her parents found her lying on the floor, immobile.

Before the onset of her psychiatric symptoms, Ms. C had been high functioning. She had been an athlete in college and had a career as a school psychologist. The Sidebar (page 26) summarizes Ms. C's psychiatric history, which includes similar complex episodes and multiple hospitalizations. She also has a history of hypothyroidism.

In the ED, the psychiatry team evaluates Ms. C. She displays a similar pattern of mutism, immobility, and rigidity as she did upon her initial presentation. Her father reports that she had been compliant with her medications but had not taken them the previous night. Ms. C screens positive for catatonia on the Bush-Francis Catatonia Rating Scale (BFCRS). Her severity score of $10 / 69$ indicates a mild presentation. She is diagnosed with catatonia and is administered IV lorazepam, $2 \mathrm{mg}$, with no response.

Because Ms. $C$ has been hospitalized many times for similar presentations, the treatment team decides to initiate a trial of IV ketamine. 
Catatonia can manifest in many different ways in patients with psychiatric illness. If left untreated, it is associated with a high rate of mortality. ${ }^{1}$ Catatonia often is described along a continuum from retarded/stuporous to excited, and presentations can vary substantially. The physiologic and psychological mechanisms of catatonia are poorly understood.

Traditionally, most patients respond well to low-dose benzodiazepines, with electroconvulsive therapy as a second-line intervention for refractory and malignant cases. However, these interventions are not always successful or readily available.

Research into the anesthetic ketamine is gradually expanding, and the use of this agent for treating various psychiatric illnesses, including both unipolar and bipolar depression, has been increasing. ${ }^{2}$ Empiric evidence suggests ketamine is effective for certain psychiatric disorders, but the mechanism of action remains unclear. Although the evidence base is small, additional cases demonstrating the effectiveness of ketamine in the treatment of acute catatonia might make it a therapeutic option for use by psychiatrists and emergency medicine clinicians.

In this article, we discuss ketamine's possible role in the treatment of catatonia, possible adverse effects, dosing strategies, and theories about ketamine's mechanism of action.

\section{Ketamine's utility in psychiatry}

Ketamine is a rapid-acting anesthetic that acts primarily by antagonizing N-methyl-Daspartate (NMDA) receptors in the CNS. It is characterized by dissociative anesthesia as it disrupts association pathways in the brain. ${ }^{3}$ Ketamine has been shown to be efficacious in treating MDD and posttraumatic stress disorder, and for the long-term management of chronic pain disorders, including fibromyalgia and many neuropathic conditions. ${ }^{4-7}$ Esketamine is an intranasal formulation of ketamine that is FDA-approved for treatment-resistant depression. ${ }^{8}$ There is scant literature describing using ketamine for treating catatonia.

Previously, ketamine had been thought to induce a catatonic state, which was supported by a neurophysiologic model of catatonia that suggested the condition was caused in part by glutamate hypoactivity at the NMDA receptor. ${ }^{9}$ However, recent studies have shown that the NMDA receptor antagonists amantadine and memantine may be useful for treatment-refractory cases of catatonia, which suggests that a broader model of glutamatergic dysfunction, and not simply glutamate hypoactivity, may be more accurate. $^{10,11}$ Denysenko et $\mathrm{al}^{12}$ proposed that the efficacy of memantine for patients with lorazepam-resistant catatonia could be explained by increases in dopamine levels in the frontal cortex and striatum. While this effect could explain why amantadine has anticatatonic effects, it does not explain why other NMDA antagonists such as ketamine are potentially pro-catatonic. Other proposed physiologic mechanisms explaining this difference include the fact that memantine and ketamine have differing effects on brain-derived neurotrophic factor (BDNF) expression and certain glutamatergic postsynaptic density proteins..$^{13,14}$

Ketamine originally was used for sedation, and much of its safety and risk profile has been developed from decades of administration as an anesthetic. Studies have found that ketamine has a large therapeutic window in children and adults. ${ }^{15,16}$ Moreover, it does not depress the respiratory system. As an anesthetic, ketamine has a rapid onset and a quick resolution, with its sedative and disorienting effects resolving within 30 to 120 minutes. ${ }^{17}$ Ketamine's rapid onset of action extends beyond its sedating effects. Trials with the intranasal spray esketamine for treatment-resistant depression have demonstrated an onset antidepressant effects within 2 days. ${ }^{18}$ This is much faster than that of traditional antidepressants, such as selective serotonin reuptake inhibitors. ${ }^{18}$ Based on these features, ketamine has the potential to be a useful medication in the emergency psychiatric setting, particularly for acute presentations such as catatonia.

\section{Beware of the potential risks}

Although ketamine may be clinically useful, it also carries some risks. Adverse effects associated with ketamine include sedation,

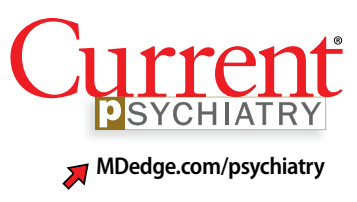

\section{Clinical Point}

\section{Empiric evidence suggests ketamine is effective for certain psychiatric disorders, but the mechanism of action remains unclear}

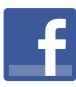

Discuss this article at www.facebook.com/ MDedgePsychiatry 


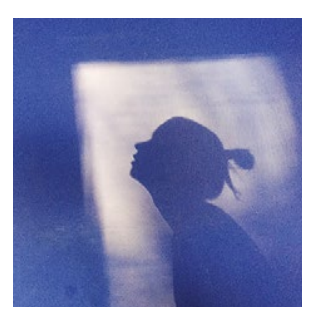

Ketamine for catatonia dissociation, hallucinations, elevated blood pressure, nausea, increased heart rate, vomiting, dizziness, fatigue, blurred vision, itching, and emesis. Clinicians also should be aware that some patients may use illicit ketamine, either as self-treatment to control depressive symptoms or for recreational purposes. When misused/abused, longterm use of ketamine can cause neurologic damage. ${ }^{19}$ Studies also have reported rare occurrences of recurrent hallucinations even after discontinuation of ketamine. ${ }^{20}$ Animal studies have demonstrated addiction and cognitive deficits with repeated use of ketamine in rodents. ${ }^{21}$ This research has led to concerns that chronic use of ketamine to treat illnesses such as depression might lead to similar long-term adverse outcomes.

\section{Dosing}

As a sedative, IV ketamine dosing is generally 1 to $2 \mathrm{mg} / \mathrm{kg}$, and IM ketamine dosing is 3 to $5 \mathrm{mg} / \mathrm{kg}$. ${ }^{16} \mathrm{As}$ an antidepressant, small

\section{SIDEBAR}

\section{Ms. C's psychiatric history}

In 2013, Ms. C experienced severe social stress from both her work as a psychologist and a divorce. She sold all of her possessions and was living in motels and hotels searching for the "truth of God." In February 2016, she was hospitalized after refusing to eat and self-discontinuing all medications, including her thyroid medications. She was then placed under the conservatorship of her parents.

In July 2017, Ms. C was hospitalized again for refusing to eat or take her medications; this time she also exhibited selective mutism. Catatonia was suspected and she was started on oral lorazepam, $2 \mathrm{mg} 3$ times a day. Duloxetine and ziprasidone were also trialed but were stopped due to noncompliance and adverse effects. Ms. C showed little improvement on these regimens. In the hospital, IV lorazepam, $4 \mathrm{mg}$, was trialed with good effect, and she began to respond to questioning. She was transitioned to oral lorazepam, $4 \mathrm{mg} 5$ times per day, and mirtazapine, $15 \mathrm{mg} / \mathrm{d}$. With this regimen, Ms. C became progressively more interactive; however, she still refused to eat. Throughout her hospitalization, multiple medications were prescribed, including divalproex sodium, memantine, zolpidem, olanzapine, and dextroamphetamine/levoamphetamine, all of which were not effective in stimulating her appetite. Due to malnutrition, Ms. C was placed on total parenteral nutrition.
During this time, the highest dose of IM lorazepam was $20 \mathrm{mg} / \mathrm{d}$ in divided doses.

\section{Some improvement with ECT}

Four months into her hospitalization, Ms. C's lorazepam was titrated down to $4 \mathrm{mg} 4$ times a day, and she underwent a trial of electroconvulsive therapy (ECT). Following the fourth ECT session, she displayed significant improvement. Ms. C engaged with her clinicians, displayed bright mood and affect, began eating again, and was able to recount her depressive symptoms following her divorce. At this time, she received a total of 8 ECT treatments and was started on fluoxetine. At the end of January 2018, after 19 days of hospitalization, she was transitioned to a partial hospitalization program (PHP) on a regimen of lorazepam, $2 \mathrm{mg} 3$ times daily; fluoxetine, $40 \mathrm{mg} / \mathrm{d}$; midodrine, $10 \mathrm{mg} 3$ times daily; fludrocortisone; and levothyroxine. Her discharge diagnosis was major depressive disorder with psychotic features and catatonia.

Between her first hospitalization and her current presentation to the emergency department (ED), Ms. C presented several times to the ED with similar symptoms of decreased speech, movement, and oral intake. In February 2018, she was hospitalized and responded after 4 sessions of ECT. She returned to work as a substitute teacher and was stable for $>1$ year on a regimen of lorazepam, olanzapine, and risperidone. In June 2019, her symptoms returned. She was hospitalized and required a nasogastric tube to address malnutrition. She was eventually stabilized on a regimen of risperidone and lorazepam, which she continued as an outpatient until she was hospitalized again in August 2019. During this hospitalization, Ms. C failed to respond to risperidone or lorazepam, up to $2 \mathrm{mg}$ 3 times a day. After several changes to her regimen, she began to respond to olanzapine, $30 \mathrm{mg} / \mathrm{d}$; mirtazapine, $15 \mathrm{mg} / \mathrm{d}$; and lorazepam, $2 \mathrm{mg} 3$ times a day.

Throughout her hospitalizations, once she became verbal, Ms. C demonstrated hyper-religiosity. She would ask to read the Bible, and state that her purpose was to find the truth of God. As an outpatient, she would compulsively go to church in the middle of the night and read the Bible for hours. A preliminary diagnosis of obsessive-compulsive disorder was made based on her scrupulosity, and mirtazapine was cross-titrated to fluvoxamine prior to discharge.

Shortly after discharge, she was readmitted to a PHP, and did well on fluvoxamine, $100 \mathrm{mg}$ twice a day; olanzapine, $5 \mathrm{mg}$ every night; levothyroxine, $100 \mathrm{mcg} / \mathrm{d}$; and oral lorazepam, $1 \mathrm{mg} 4$ times a day. Ms. $C$ displayed full mood, appropriate affect, and began working part-time as a substitute teacher. She had begun to interview for full-time jobs before her most recent ED presentation. 
clinical trials have suggested that the preferred dose of IV ketamine may be 0.5 to $1 \mathrm{mg} / \mathrm{kg}$, with dose-dependent increases in dissociation and blood pressure. ${ }^{21}$ Studies have also demonstrated that oncedaily IV ketamine, $0.5 \mathrm{mg} / \mathrm{kg}$ administered over 40 minutes, led to greater improvements in patients with MDD than placebo, whereas once-daily IV ketamine, $0.2 \mathrm{mg} / \mathrm{kg}$, did not. ${ }^{20}$

\section{CASE CONTINUED}

The team begins to treat Ms. C with IV ketamine. Ketamine, $0.2 \mathrm{mg} / \mathrm{kg}$, is used to calculate the initial dose, and a total of $10 \mathrm{mg}$ is administered over 10 minutes. Fifteen minutes after administration, Ms. C is able to move around in her bed, make eye contact, and nod to questions. She has purposeful movements, such as examining her IV line, scratching her head, and repositioning herself in the bed. After a few more minutes, she makes eye contact with her father, and nods to him during conversation. She is able to make a few noises but does not speak.

Later that day, Ms. C is discharged home (in a wheelchair) with her parents, on a medication regimen of fluvoxamine, $100 \mathrm{mg} / \mathrm{d}$; lorazepam, $1 \mathrm{mg} 4$ times a day; and olanzapine, 5 $\mathrm{mg} / \mathrm{d}$. She is scheduled for an outpatient follow-up appointment 5 days later. Her parents are given instructions and several precautions to ensure that Ms. C receives proper nutrition until her appointment. That evening, Ms. $C$ is able to eat voluntarily.

Five days later, Ms. C visits the outpatient psychiatric clinic and is verbal and ambulatory. Her father reports that she has become more verbal. During her follow-up interview, she is observed to be more subdued and less verbal than her baseline, but is vocal and able to voice her understanding of the treatment plan.

After 3 months of being stable on her outpatient regimen, Ms. C's catatonic symptoms return, including refusing to eat and mutism. She is administered IV lorazepam, $4 \mathrm{mg}$, with no response and is admitted to the hospital for placement of a nasogastric feeding tube to address malnutrition. After several days, Ms. $C$ responds to lorazepam, $4 \mathrm{mg}$ every 6 hours. Six days later, after she begins eating and taking her medications voluntarily and the nasogastric tube is removed, Ms. C is discharged to home.

\section{Findings need to be replicated in larger studies}

Although some research has indicated that ketamine may be pro-catatonic, Ms. C's improvement after receiving ketamine suggests that perhaps the situation is more complex. ${ }^{12,22}$ The exact mechanisms underlying catatonia remain uncertain. Carroll et $\mathrm{al}^{9}$ described 4 theories, and only 1 of them involved glutamate. Additionally, ketamine's mechanism of action may extend beyond NMDA antagonism. In our case, Ms. C's low BFCRS score during her most recent visit to the ED suggests she may have had a milder or less typical form of catatonia compared with her previous presentations (Sidebar, page 26). However, Ms. C's clinical improvement after receiving ketamine is noteworthy.

A review of the literature yielded only 1 other case report that described using ketamine to treat catatonia. ${ }^{23}$ Iserson et $\mathrm{al}^{23}$ reported that their patient's catatonic symptoms resolved after a total of $12.5 \mathrm{mg}$ of ketamine was administered in $0.03 \mathrm{mg} / \mathrm{kg}$ boluses every 3 minutes. Compared with our own protocol, ketamine was administered at a much slower rate in this case, although both total doses of ketamine were comparable and well below the dose used for sedation. Additionally, in Iserson et $\mathrm{al}^{23}$ lorazepam was not administered before ketamine because lorazepam was not readily available in the treatment setting. In our case, Ms. C may have had a delayed response to the IV lorazepam she received an hour before the ketamine dose; however, she exhibited a distinct clinical improvement 10 to 15 minutes after IV ketamine was administered. Nevertheless, both cases demonstrated rapid resolution of catatonic symptoms following administration of ketamine.

The marked improvement after the ketamine infusion allowed Ms. C to be discharged from the ED the same day, which was never possible after her previous catatonic episodes. Five days after discharge, she was walking, eating, talking,

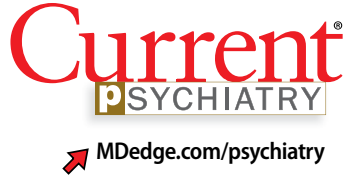

Clinical Point

In 1 other case report, a patient's symptoms of catatonia resolved after receiving IV ketamine, $12.5 \mathrm{mg}$ 


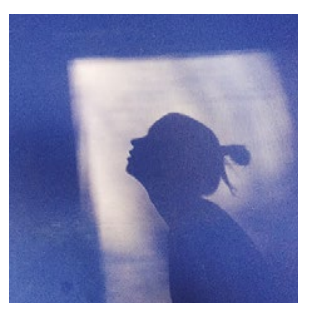

Ketamine for catatonia

\section{Related Resources}

- Dubovsky SL, Dubovsky AN. Catatonia: How to identify and treat it. Current Psychiatry. 2018;17(8):16-26.

- Iserson KV, Durga D. Catatonia-like syndrome treated with low-dose ketamine. J Emerg Med. 2020;58(5):771-774.

Drug Brand Names

Amantadine - Gocovri Dextroamphetamine sulfate/levoamphetamine sulfate - Evekeo

Divalproex sodium .

Depakote

Duloxetine - Cymbalta

Esketamine - Spravato

Fluoxetine - Prozac

Fludrocortisone - Florinef
Fluvoxamine - Luvox Ketamine $\cdot$ Ketalar Levothyroxine - Synthroid Lorazepam - Ativan Memantine - Namenda Mirtazapine $\cdot$ Remeron Olanzapine - Zyprexa Risperidone - Risperdal Ziprasidone - Geodon Zolpidem - Ambien

\section{Clinical Point}

Our patient's catatonic symptoms improved 10 to 15 minutes after receiving ketamine, and she remained stable for 5 months and able to attend to her activities of daily living without any change to her other medications. Moreover, these effects outlasted the duration of ketamine. Ms. C remained stable for 5 months until she destabilized in June 2020. At that time, she did not respond to lorazepam in the ED, needed to be hospitalized, and required a nasogastric feeding tube. Ketamine was not trialed during this presentation, so it remains to be seen if the patient's response to ketamine was an isolated incident, or whether it could potentially spare her from future hospitalizations.

\section{References}

1. Rasmussen SA, Mazurek MF, Rosebush PI. Catatonia: our current understanding of its diagnosis, treatment and pathophysiology. World J Psychiatry. 2016;6(4):391-398.

2. Grady SE, Marsh TA, Tenhouse A, et al. Ketamine for the treatment of major depressive disorder and bipolar depression: a review of the literature. Mental Health Clin. 2017;7(1):16-23.

3. KETALAR (ketamine hydrochloride) injection. (n.d.). Accessed April 29, 2021. https://www.accessdata.fda.gov/ drugsatfda_docs/label/2017/016812s043lbl.pdf

4. Williams NR, Schatzberg AF. NMDA antagonist treatment of depression. Curr Opin Neurobiol. 2016;36:112-117.

5. Parashchanka A, Schelfout S, Coppens M. Role of novel drugs in sedation outside the operating room: dexmedetomidine, ketamine and remifentanil. Curr Opin Anaesthesiol. 2014;27(4):442-447.
6. Radvansky BM, Puri S, Sifonios AN, et al. Ketamine-a narrative review of its uses in medicine. Am $\mathrm{J}$ Ther. 2016;23(6):e1414-e1426. doi: 10.1097/MJT.0000000000000257

7. O'Brien SL, Pangarkar S, Prager J. The use of ketamine in neuropathic pain. Current Physical Medicine and Rehabilitation Reports. 2014;2(2):128-145.

8. Swainson J, Thomas RK, Archer S, et al. Esketamine for treatment resistant depression. Expert Rev Neurother. 2019;19(10):899-911.

9. Carroll BT. The universal field hypothesis of catatonia and neuroleptic malignant syndrome. CNS Spectr. 2000;5(7): 26-33.

10. Carroll BT, Goforth HW, Thomas C, et al. Review of adjunctive glutamate antagonist therapy in the treatment of catatonic syndromes. J Neuropsychiatry Clin Neurosci. 2007;19(4):406-412.

11. Northoff G, Eckert J, Fritze J. Glutamatergic dysfunction in catatonia? Successful treatment of three acute akinetic catatonic patients with the NMDA antagonist amantadine. J Neurol Neurosurg Psychiatry. 1997;62(4):404-406.

12. Denysenko L, Sica N, Penders TM, et al. Catatonia in the medically ill: etiology, diagnosis, and treatment. The Academy of Consultation-Liaison Psychiatry EvidenceBased Medicine Subcommittee Monograph. Ann Clin Psychiatry. 2018;30(2):140-155.

13. Gideons ES, Kavalali ET, Monteggia LM. Mechanisms underlying differential effectiveness of memantine and ketamine in rapid antidepressant responses. Proc Natl Acad Sci U S A. 2014;111(23):8649-8654.

14. de Bartolomeis A, Sarappa C, Buonaguro EF, et al. Different effects of the NMDA receptor antagonists ketamine, MK801 , and memantine on postsynaptic density transcripts and their topography: role of Homer signaling, and implications for novel antipsychotic and pro-cognitive targets in psychosis. Prog Neuropsychopharmacol Biol Psychiatry. 2013;46:1-12.

15. Green SM, Johnson NE. Ketamine sedation for pediatric procedures: part 2, review and implications. Ann Emerg Med. 1990;19(9):1033-1046.

16. Kurdi MS, Theerth KA, Deva RS. Ketamine: current applications in anesthesia, pain, and critical care. Anesth Essays Res. 2014;8(3):283-290.

17. Majidi S, Parna A, Zamani M, et al. Onset and effect duration of intrabuccal space and intramuscular ketamine in pediatrics. Adv Biomed Res. 2018;7:91.

18. Bahr R, Lopez A, Rey JA. Intranasal esketamine (SpravatoTM) for use in treatment-resistant depression in conjunction with an oral antidepressant. P T. 2019; 44(6):340-342,344-346,375

19. Strong CE, Kabbaj M. On the safety of repeated ketamine infusions for the treatment of depression: effects of sex and developmental periods. Neurobiol Stress. 2018;9:166-175.

20. Su TP, Chen MH, Li CT, et al. Dose-related effects of adjunctive ketamine in Taiwanese patients with treatmentresistant depression. Neuropsychopharmacology. 2017; 42(13):2482-2492.

21. Fava M, Freeman MP, Flynn M, et al. Double-blind, placebocontrolled, dose-ranging trial of intravenous ketamine as adjunctive therapy in treatment-resistant depression (TRD). Mol Psychiatry. 2020;25(7):1592-1603.

22. Wong DH, Jenkins LC. An experimental study of the mechanism of action of ketamine on the central nervous system. Can Anaesth Soc J. 1974;21(1):57-67.

23. Iserson $\mathrm{KV}$, Durga $\mathrm{D}$. Catatonia-like syndrome treated with low-dose ketamine. J Emerg Med. 2020;58(5): 771-774.

\section{Bottom Line}

In our case report, a woman with a long history of catatonia responded to a single infusion of IV ketamine, and the beneficial effects lasted for months. More research evaluating the efficacy of ketamine is needed to determine if this agent has a place in the treatment of catatonia. 